\title{
4
}

\section{Pressure Surges Following Sudden Air Pocket Entrapment in Stormwater Systems}

\author{
Jose G. Vasconcelos and Gabriel M. Leite
}

Stormwater storage tunnels may undergo rapid pipe filling conditions during extreme rain events. Such conditions are relevant as adverse conditions may develop, such as surging due to entrapment and compression of entrapped air pockets. Operational issues such as structural damage, geysering and the return of conveyed water to grade, among others, have been linked to air pocket entrapment.

This chapter presents results from the experimental investigations of pressure surges caused by sudden air pocket entrapment. Steady flow was supplied in a pipeline in such a way that pressurized flows existed at the upstream end while the downstream end experienced free surface flow due to free discharge conditions at the downstream end. A combination of flow rates and slopes resulted in several gradually varied flow profiles at the discharge, with various volumes of atmospheric air at the discharge end.

By suddenly closing the downstream discharge valve, an air pocket was entrapped and surges were recorded. To emulate conditions in which surge relief is provided during air pocket compression, valve operation also included cases with partial valve obstruction. Among the obtained results, one can notice significantly different surges for cases with and without pressure relief (e.g. with total or partial obstruction), and that the larger the obstruction degree the larger are the surges. Such findings are useful in the development of numerical models to simulate the rapid filling of stormwater tunnels incorporating these findings.

Vasconcelos, J. and G.M. Leite. 2012. "Pressure Surges Following Sudden Air Pocket Entrapment in Stormwater Systems." Journal of Water Management Modeling R245-04. doi: 10.14796/JWMM.R245-04.

(C) CHI 2012 www.chijournal.org ISSN: 2292-6062 (Formerly in On Modeling Urban Water Systems. ISBN: 978-0-9808853-7-8) 


\subsection{Introduction}

Stormwater storage tunnels experience extreme flow conditions during intense rain events, when rapidly filling pipe conditions exist. Such conditions are relevant to designers because large pressure surges may occur as a result of the compression of air pockets that may be entrapped during the rapid filling of those conduits. Reports in the literature indicate operational issues linked with pressure surges, such as structural damage (Zhou, et al. 2002a), geysering and the return of conveyed water to grade (Guo and Song, 1991; Wright, et al., 2011); entrapped air pockets may also lead to a significant loss of storage volume. Such operational issues have implications in public safety and public health, as well as significant material costs.

Past investigations have studied mechanisms leading to air pocket entrapment during the rapid filling of closed conduits. Hamam and McCorquodale (1982) and Li and McCorquodale (1999) investigated pockets formed by shear flow instabilities due to the relative motion of the air and water phases. Zhou et al. (2002b) investigated air pockets entrapped during the rapid filling of conduits due to limited ventilation. Vasconcelos and Wright (2005) and Vasconcelos and Wright (2006) presented systematic studies on the filling of a nearhorizontal pipeline with varying ventilation configurations, and identified other mechanisms for air pocket entrapment such as the interface breakdown mechanism. Leite and Vasconcelos (2011) studied the compression of an air pocket by a backward moving inflow front in the context of the rapid filling of sloped water mains.

Nonetheless, investigations of pressure surges following the entrapment of air pockets in stormwater systems have been limited. Possibly one of the first contributions to this topic is the work by Martin (1976) which considered the compression of an air pocket against the downstream end of a capped, upslope pipeline following the opening of a valve at the upstream, separating the pipeline from a reservoir with a higher piezometric head. It was shown that the peak pressure head was much larger than the reservoir head at the upstream end, and these surge peaks increased for smaller air pockets.

A similar experimental study on the filling of sloped pipelines performed by Fuertes et al. (2000) confirmed this behaviour when air pockets were trapped between adjacent water masses. Zhou et al. (2002a) studied the entrapment, compression and release of air pockets in systems ventilated through orifices. The authors pointed large pressure surges during air pocket compression and expansion cycles, and a stronger water hammer pulse when the pocket was completely expelled from the system.

One relevant limitation on the previous studies is that the conditions in which those pockets were entrapped may be too extreme to be representative of 
those expected in stormwater systems. In some cases, the ratio between the driving head and pipe diameter was over 100. If Reynolds scaling is used to represent this pressurization front in prototype scale, this would correspond to the advance of a pipe filling bore front compressing an air pocket in a $2 \mathrm{~m}$ diameter conduit with a driving pressure head above $200 \mathrm{~m}$ water. Clearly, these extreme conditions are not representative of situations expected in stormwater tunnels in which air pockets become entrapped and compressed. An instance is when air pocket entrapment occurs by the reflection of flow fronts within the system's physical boundaries (such as vertical shafts), a condition that has not been sufficiently investigated to date.

Accurate modeling of rapid filling in stormwater tunnel filling has indicated that the interaction of open channel bores at upstream boundaries (e.g. reflection at dropshafts) may result in pocket entrapment, a mechanism which was also predicted by Vasconcelos and Wright (2006). This is shown in Figure 4.1.

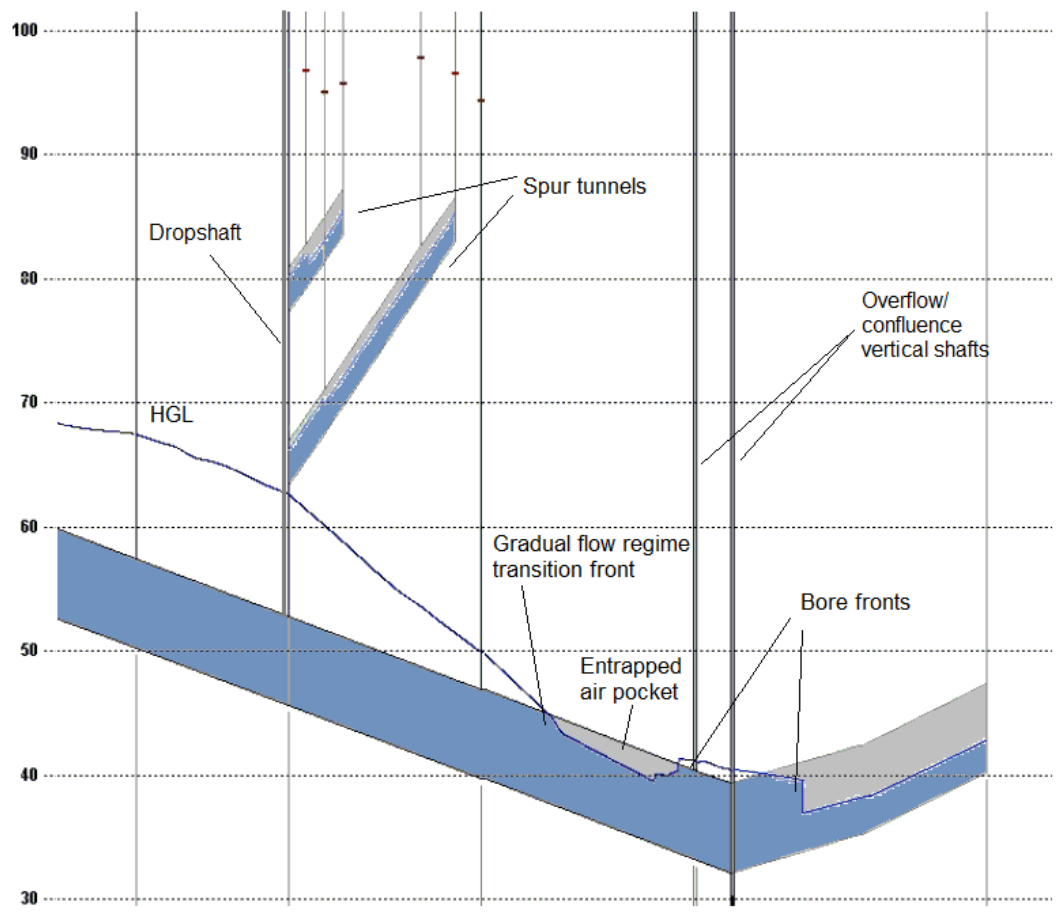

Figure 4.1 Air pocket entrapment predicted by numerical modeling; pocket entrapment occurs as inflow accumulates at the lowest point, between the flow regime transition front and a backward moving bore. 
As exemplified in the numerical simulation results presented in Figure 4.1, an initial open channel bore advances towards a dropshaft, and after reaching that point, it is possible that the water level at that location increases to a point where the reflected bore becomes a pipe filling bore. Ahead of the backward moving bore, an air pocket is formed, while the general flow direction in the conduit may still be directed towards the shaft due to the system inertia. Air phase pressure is expected to rise, but the presence of the nearby shafts may significantly attenuate pressure surges. No experimental study to date has addressed such a situation, and to neglect the attenuation caused by the nearby shaft will lead to significant pressure over-prediction.

Most experimental studies on surging caused by air compression involving complete valve closure (Martin, 1976; Zhou et al. 2002; De Martino et al., 2008), creating conditions that not conducive for surging as air pockets are compressed by the inflow without any relief (e.g. air release). On experiments involving air release by orifices, surging was smaller but one question is whether this relief mechanism is representative of what would be expected in tunnel systems. In actual tunnels relief is provided by water storage in shafts, much like the effect of a standpipe in a pipeline, and not by release of air.

\subsection{Knowledge Gaps and Objectives}

Some of the relevant knowledge gaps related to air pocket entrapment and compression in stormwater tunnel systems are discussed in this section.

Air pocket pressurization has not been investigated when inflow interaction with system boundaries (e.g. bore reflection) is the mechanism behind pocket formation. Such cases are not expected to be as extreme as the conditions that have been considered in previous studies (e.g. Martin, 1976; Zhou et al., 2002a) because of the pressure relief that shafts may provide. They are very relevant as they may result in the loss of tunnel storage volumes. Of particular interest is the magnitude of the surges upon pocket entrapment and whether in such cases negative pressures will be observed.

Air pocket compression has not been sufficiently studied in pressure ranges that are comparable to the expected conditions in stormwater tunnels. In most previous studies the ratio between the driving pressure head of the flow and the conduit diameter was excessive, much larger than those expected in a stormwater tunnel system undergoing rapid filling. One questions how representative were such previous studies in representing surges in actual stormwater tunnels.

It is expected that rapid flows will generate larger surges upon blockage; larger surges are also expected when smaller air pockets are compressed (Martin, 1976). To date no study has considered these two factors separately, particularly in the context of air pocket entrapment. This may make difficult the 
development and calibration of numerical models to simulate tunnel filling events taking account of air pressurization effects.

This chapter presents the experimental results from an ongoing investigation, at Auburn University, into surges caused by air pocket entrapment emulating the mechanism of bore reflection. The idea is to measure the magnitude of the pressure surges for varying air pocket volumes, system slopes, initial flow rates and obstruction levels caused by a discharge valve. By varying the obstruction caused by valves we aim to emulate conditions in which water removal causes surge mitigation. It is assumed that the gate would create conditions similar to rising water at shafts, decreasing, but not zeroing, the outflow into the shafts during the air pocket compression process. By investigating this particular flow condition, the main goal is to gain better insight into the underlying physics and thus improve current modeling approaches to the problem by better incorporating air pressurization effects.

\subsection{Methodology}

The experimental apparatus used in the experiments, shown in Figure 4.2, is essentially two reservoirs connected by a clear PVC pipeline in a recirculating system. The upstream reservoir volume is $0.624 \mathrm{~m}^{3}$ and the downstream reservoir volume is $0.662 \mathrm{~m}^{3}$. The pipeline length and diameter are $12.0 \mathrm{~m}$ and 0.101 $6 \mathrm{~m}$ respectively. The upstream reservoir has a $50 \mathrm{~mm}$ tap that expands into the pipeline diameter. At the downstream end a PVC knife gate valve of $0.1016 \mathrm{~m}$ diameter controls the outflow, which discharges freely into the downstream reservoir when the valve is open.

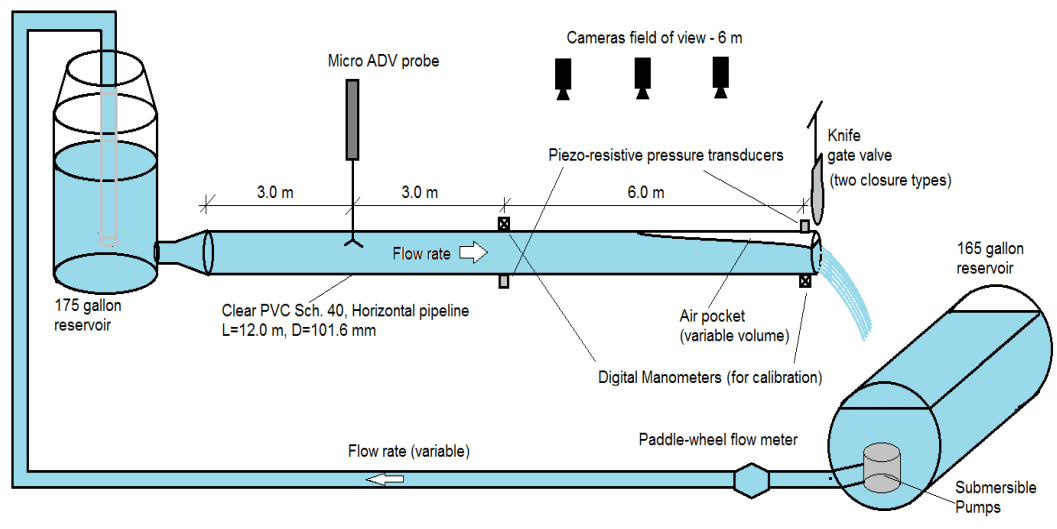

Figure 4.2 Experimental apparatus used to perform the investigations on air pocket entrapment and compression. 
The experimental instruments include three digital camcorders, a Nortek Vectrino MicroADV probe, two ENDEVCO 8510 C-50 pressure transducers, and a paddlewheel flow meter.

The experiments performed to this date have used horizontal and adverse slopes as a means of gaining better understanding of the interactions following the gate valve operations. Up to five different flow rates were used, with larger flow rates yielding smaller air pockets at the pipeline downstream end. Various air pockets were created due to the existing drawdown profiles caused by the free discharge (gradually varied flow), which also depended on the pipe slope. For the initial experiments, two different methods were used to restrain the pipes: anchoring from the upstream end only or anchoring from both ends of the apparatus, but eventually the dual anchor system was used in all remaining experiments.

Three different levels of obstruction were used for the knife gate: $100 \%$ obstruction, $81 \%$ area obstruction (bottom clearance of $13 \mathrm{~mm}$ from the knife gate valve seat) and $89 \%$ area obstruction (bottom clearance of $8 \mathrm{~mm}$ ). For the partial obstruction condition, four different flow rates were tested.

The experimental procedure in the experiments followed was:

1. With the selected slope set in the pipeline, the pumps were started and correspondent flow valves were opened, feeding the upstream reservoir creating steady flow conditions in the clear PVC pipeline;

2. The flow rate was chosen so that the upstream end of the pipeline was in a pressurized regime;

3. The initial pressures at both manometers were read, along with the head at the upstream reservoir;

4. Air pocket volumes were calculated by measuring the dry perimeter at the clear PVC pipe crown, caused by the air cavity, at regular intervals prior to gate operation; interval sizes ranged between $0.05 \mathrm{~m}$ for the smallest air pockets to $0.30 \mathrm{~m}$ for the largest pockets;

5. With the digital cameras on, pressure measurement with the transducers (frequency $100 \mathrm{~Hz}$ ) and velocity measurement with the MicroADV probe (frequency $25 \mathrm{~Hz}$ ) commenced;

6. The flow was obstructed (partially or completely) and visual observations and remarks were made to aid later camera data analysis;

7. The pump flow was stopped, valves closed and pressures reached an equilibrium level; and

8. The final pressure levels at the manometers were read to calibrate the results from pressure transducers 
The experimental program included the experimental variables presented in Table 4.1

Table 4.1 Experimental variables and tested range.

\begin{tabular}{|c|c|c|}
\hline Variables & Tested range & Normalized range \\
\hline Flow rate $(Q)$ & $2.08 \mathrm{~L} / \mathrm{s}$ to $5.45 \mathrm{~L} / \mathrm{s}$ & $Q^{*}=Q /\left(g D^{5}\right)^{0.5}=0.202$ to 0.532 \\
\hline Slope $\left(S_{o}\right)$ & Horizontal, $2.7 \%$ and $1.3 \%$ adverse* & $\mathrm{n} / \mathrm{a}$ \\
\hline Valve obstruction & $\begin{array}{c}0 \mathrm{~mm}, 8 \mathrm{~mm} \text { and } 13 \mathrm{~mm} \text { residual } \\
\text { opening at the knife gate valve }\end{array}$ & $\begin{array}{l}100 \%, 89 \% \text { and } 81 \% \text { of pipe } \\
\text { cross section obstruction }\end{array}$ \\
\hline
\end{tabular}

* Adverse slope: slopes upward from the supply reservoir, so the pipeline discharge invert is at a higher elevation

Over 88 experimental runs were conducted, representing 40 different experimental configurations. Most experimental runs were performed in duples, with a few cases requiring triples to ensure consistency. Results presented in the next section are grouped by the degree of valve obstruction.

\subsection{Experimental Results}

\subsubsection{Total Obstruction Experiments}

Unlike experiments with partial valve obstruction, presented below, these experimental cases yielded significant down surges and sub-atmospheric pressures. An oscillatory like pattern for the pressures and flow velocity measurements was observed, with oscillation periods depending on the entrapped air pocket volume. A sequence of still images following a total obstruction operation is presented in Figure 4.3 overleaf for a horizontal slope case.

\section{Pressure Measurements}

Pressure measurements at the upstream transducer $\left(X^{*}=X / L=0.5\right)$ and at the downstream transducer $\left(X^{*}=X / L=1.0\right)$ are presented in Figures 4.4 and 4.5 below, grouped by the flow rate/initial air pocket volume for the horizontal slope, and including all repetitions.

In general, over time a sudden rise in pressure was followed by an oscillation pattern in the pressure as the incoming flow decelerates due to valve closure. Time is normalized by the factor $L /(g . D)^{0.5}$. One may notice that the pressure peaks increase for smaller air pockets, as predicted by Martin (1976). The oscillation period also increased with entrapped pocket volume, and one notices that the magnitude of the oscillations was strongly damped at each oscillation cycle. 
$T=01 \mathrm{~m} \cdot 33.35 \mathrm{~s}-$ System initial steady condition

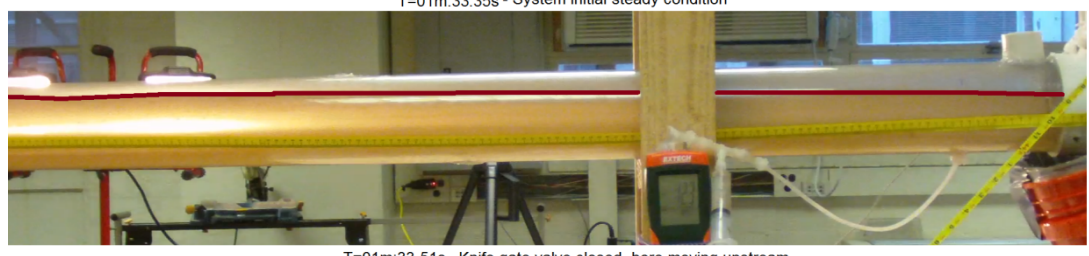

$T=01 \mathrm{~m}: 33.51 \mathrm{~s}-$ Knife gate valve closed, bore moving upstream

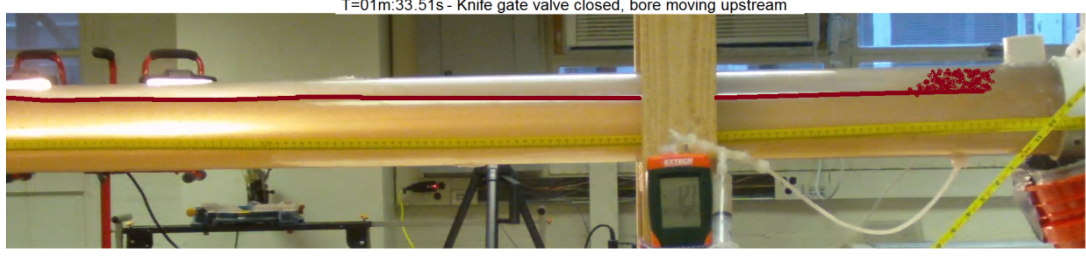

$\mathrm{T}=01 \mathrm{~m} \cdot 33.78 \mathrm{~s}$ - Pipe filling bore moving upstream

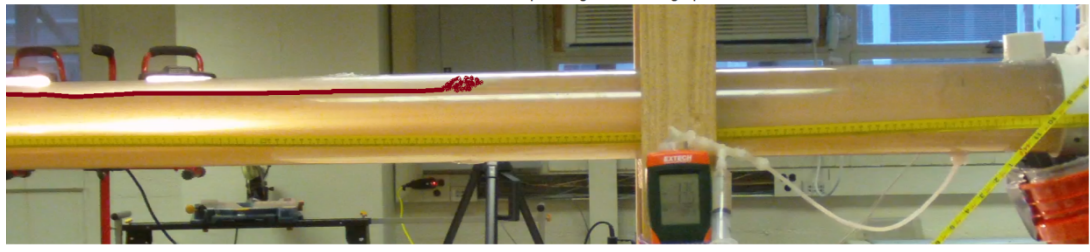

$\mathrm{T}=01: 34.11 \mathrm{~s}-$ Bore swept through the camera field of view

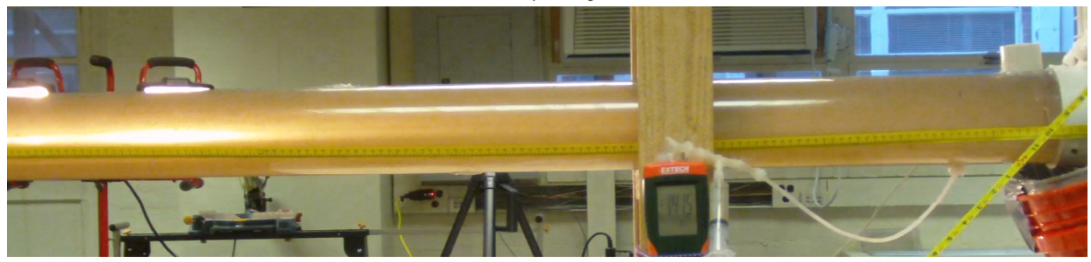

$T=01 \mathrm{~m}: 35.95 \mathrm{~s}$ - Discrete pockets moving toward valve

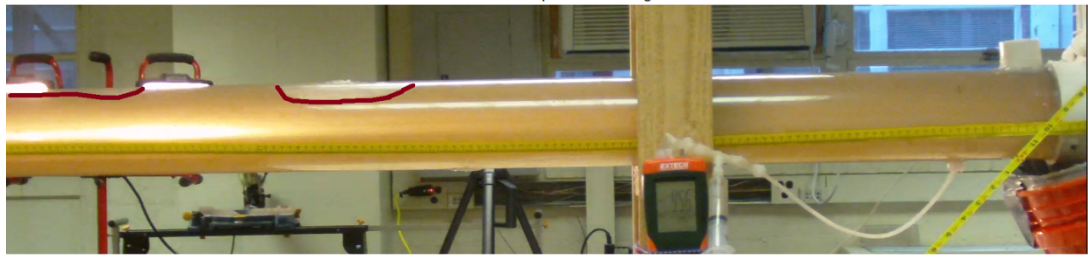

$\mathrm{T}=01 \mathrm{~m}: 39.21 \mathrm{~s}-$ Stratified conditions with no flow

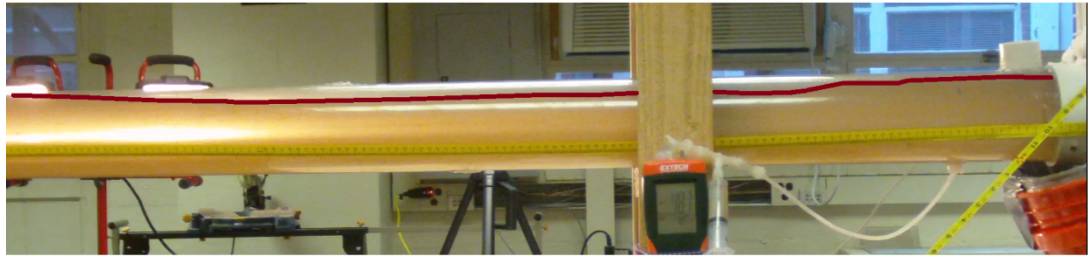

Figure 4.3 Sequence of a complete valve closure, air pocket formation and motion $\left(Q^{*}=0.44, V_{\text {air }}{ }^{*}=2.3\right.$, horizontal slope; the interface between air water phases has been artificially enhanced). 


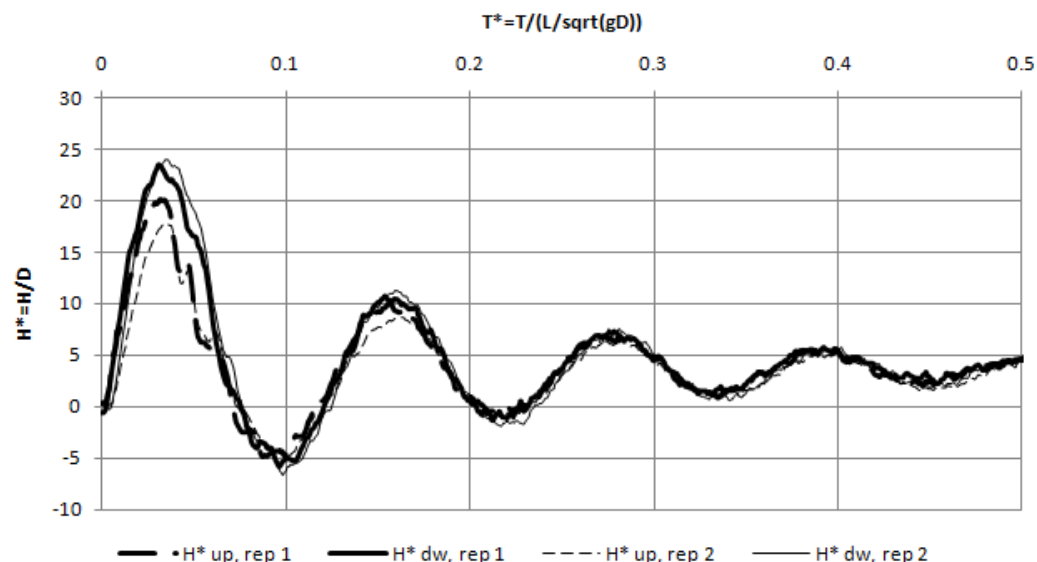

Figure 4.4 Non-dimensional pressure development following complete obstruction of the knife gate valve ( $Q=0.38-0.40, V_{\text {air }}{ }^{*}=3.4$, horizontal slope $)$; subscripts up and including $d w$ denote upstream pressure transducer results $\left(X^{*}=0.5\right)$ and downstream pressure transducer results $\left(X^{*}=1.0\right)$ respectively.

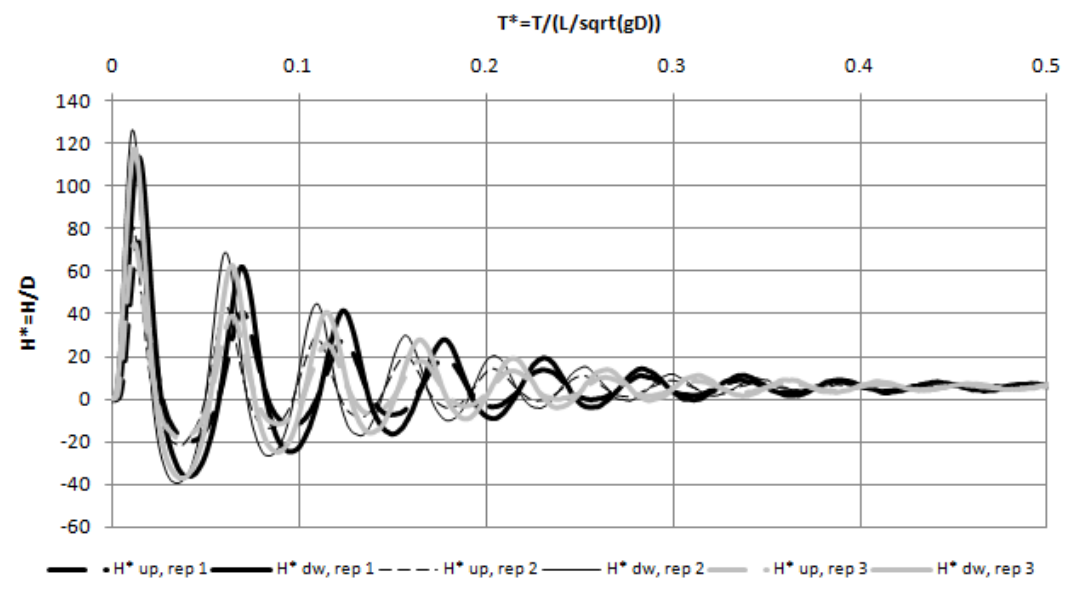

Figure 4.5 Non-dimensional pressure development following complete obstruction of the knife gate valve $\left(Q^{*}=0.51-0.52, V_{\text {air }}{ }^{*}=0.38-0.42\right.$, horizontal slope $)$. 
Visual observations of the air pocket shape upon closure suggested a very complex configuration, with the initial air intrusion breaking into several smaller pockets, and moving within the pressurized region. Discrepancies among experimental repeats are mostly likely caused by the inability to create exactly equal initial conditions prior to valve operation, specially with respect to the initial air pocket volume.

Figures 4.6 and 4.7 present comparisons between the total obstruction experiments performed at different slopes. The flow rates considered in Figure 4.6 were the largest tested for sloped conditions, and ranged between 0.49 and 0.51 .

Even though there is relatively significant variation in the air pocket volumes in that figure, in absolute terms the initial volumes of these cavities are all small, extending over a small length of the pipe crown (1.5 times to 2.5 times the pipe diameter). It is not entirely clear why peak pressures were insensitive to small variations in pocket volume, but clearly notices smaller oscillation periods with smaller entrapped air pocket volumes, as expected.

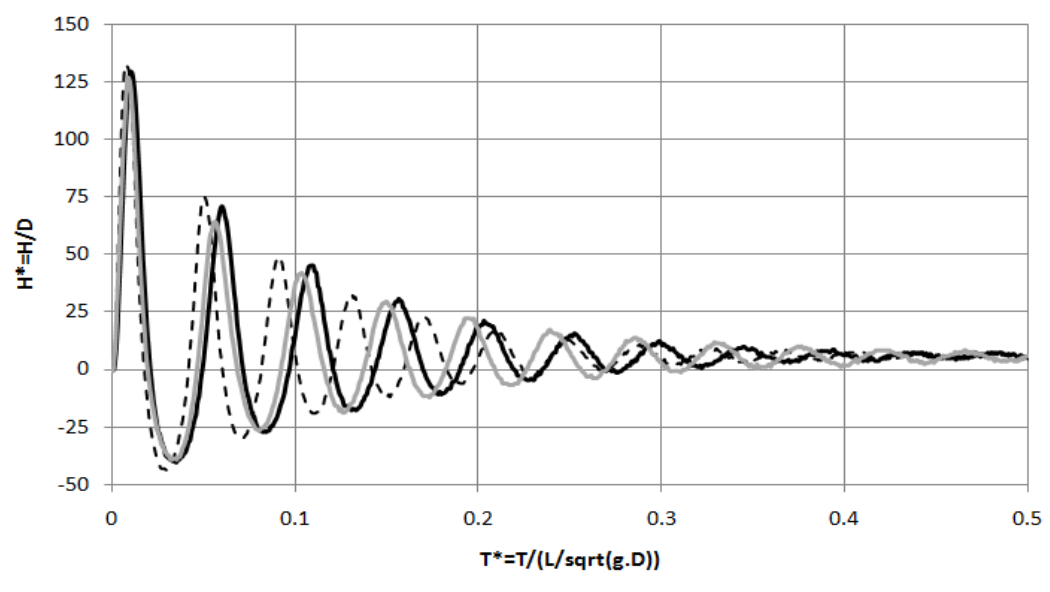

- Horizontal- Vair ${ }^{*}=0.380--1.3 \%$ adverse- Vair ${ }^{*}=0.126-2.7 \%$ adverse- Vair $*=0.245$

Figure 4.6 Non-dimensional pressure development following complete obstruction by the knife gate valve $\left(Q^{*}=0.49-0.51, V_{\text {air }}{ }^{*}=0.13-0.38\right.$, varying pipe slopes).

The results in Figure 4.7 are similar to the ones presented in Figure 4.6, except that experimental conditions were such that the flow rates were much smaller $\left(Q^{*}=0.37\right.$ to $\left.Q^{*}=0.38\right)$. 
For smaller flow rates, the varying slopes caused very different cavity volumes prior to the gate operation, with the smallest cavity volume associated with the largest pipe slope, as would be expected in a drawdown profile. Upon closure, the pressure peak was significantly higher for the case with the steepest adverse slope. The relationship between pressure peaks, air pocket volume and initial system inertia is further explored in section 4.5 below.

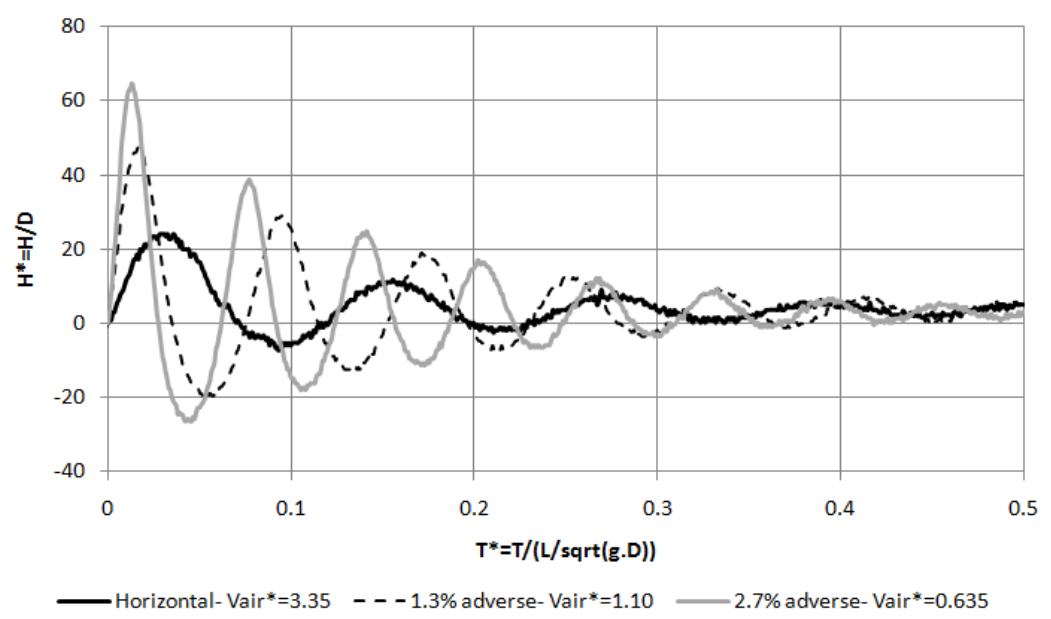

Figure 4.7 Non-dimensional pressure development following complete obstruction by the gate valve $\left(Q^{*}=0.37-\right.$ $0.38, V_{a i r}{ }^{*}=0.64-3.35$, varying pipe slopes).

\section{Flow Velocity Results}

The MicroADV probe was located $9 \mathrm{~m}$ from the knife gate valve $\left(X^{*}=0.25\right)$, and prior to the valve operation the velocity measurements were characterized by a steady velocity with expected turbulence fluctuations. As the valve was closed there was a sudden drop in the velocities, followed by the same damped oscillation pattern observed with the pressure. Results in Figure 4.8 are presented for one of each different flow rates tested.

The velocity measurements are similar to the pressure measurement results in that they also present a damped oscillatory pattern. Oscillation period was smaller for larger flow rates considering that for such conditions the entrapped air pocket volumes were smaller. Results obtained with other slopes yielded generally the same oscillatory behaviour, and are not presented. 


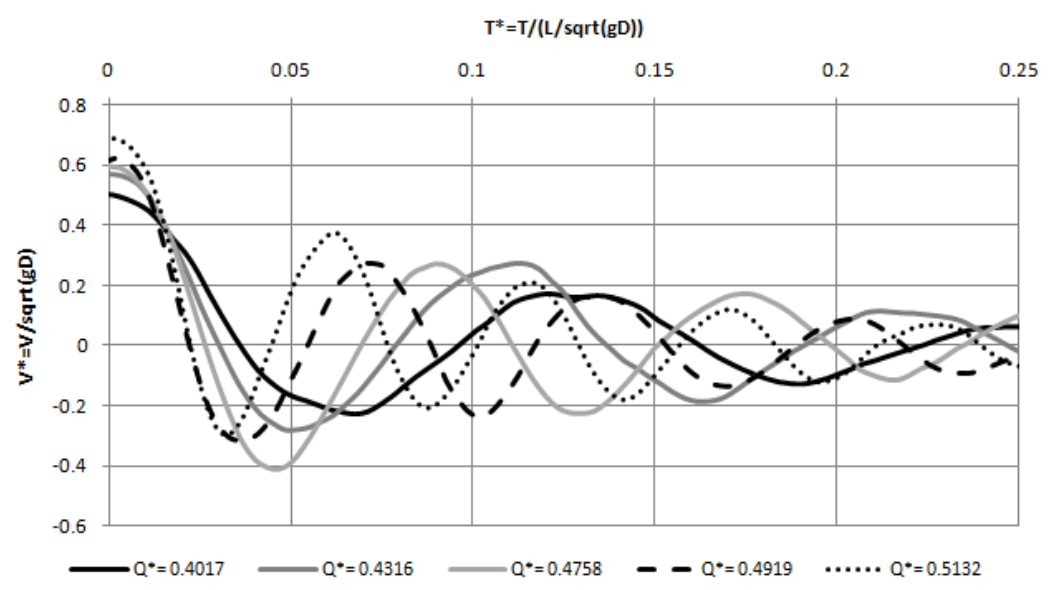

Figure 4.8 Non-dimensional velocity development following total obstruction by the knife gate valve, horizontal slope results.

\subsubsection{Partial Obstruction Experiments}

Experimental conditions involving partial obstruction of the downstream valve enforced obstructions of $81 \%$ and $89 \%$ by means of a partial obstruction of the knife gate valve. The valve was quickly closed, but rather than interrupt the flow entirely, wooden pieces blocked the motion of the valve shaft, stopping it either $13 \mathrm{~mm}(81 \%)$ or $8 \mathrm{~mm}(89 \%)$ from the valve seat.

\section{Pressure Measurements}

Figures 4.9 and 4.10 below present pressure measurement results at the downstream transducer for the partial valve obstruction for three flow rates, horizontal slope, and valve obstruction of $89 \%$ and $81 \%$ respectively.

In general the results were significantly different from those obtained with the full valve obstruction conditions. The minimum pressures were not negative, and no oscillation pattern was noticed in the recorded pressures, particularly for the $81 \%$ flow interruption. As Figure 4.9 indicates, few oscillations were noticed in some cases when $89 \%$ obstruction, yet the magnitude of those were greatly damped and rapidly dissipated.

The three flow rates represented in Figures 4.9 and 4.10 correspond to the minimum, average and maximum flow rates tested in the full obstruction experiments under horizontal slope. It is noticeable that the peak pressures are much smaller than those observed for the complete valve obstruction, as illustrated in Table 4.2 below. 


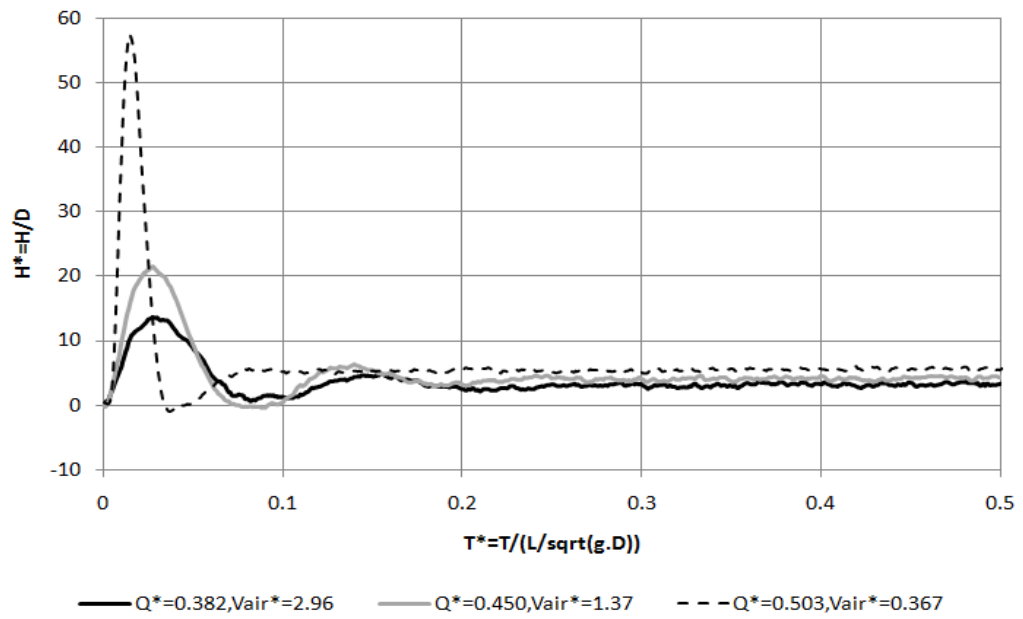

Figure 4.9 Non-dimensional pressure development following $89 \%$ obstruction by the knife gate valve $\left(Q^{*}=0.37-0.52\right.$, horizontal slope).

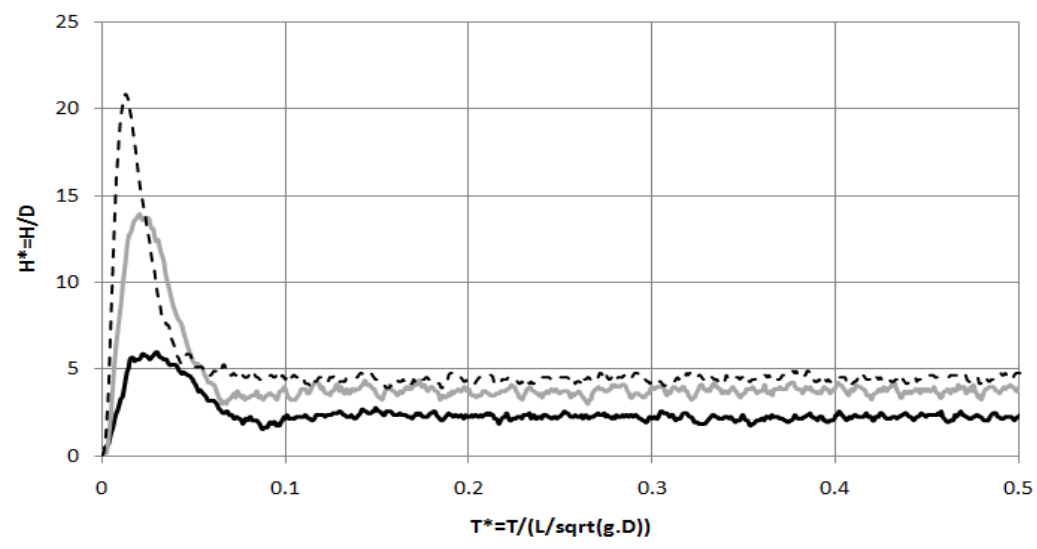

$-Q^{*}=0.371$, Vair* $=3.87$

Figure 4.10 Non-dimensional pressure development following $81 \%$ obstruction by the knife gate valve $\left(Q^{*}=0.37-\right.$ 0.52 , horizontal slope). 
Table 4.2 Comparison of peak $H^{*}=H / D$ pressures for horizontal slope experiments averaged between repetitions.

\begin{tabular}{cccc}
\hline Initial flow & Total obstruction & $89 \%$ obstruction & $81 \%$ obstruction \\
\hline$Q^{*} \approx 0.51$ & $H^{*} \approx 125$ & $H^{*} \approx 57$ & $H^{*} \approx 21$ \\
$Q^{*} \approx 0.45$ & $H^{*} \approx 57$ & $H^{*} \approx 21$ & $H^{*} \approx 13$ \\
$Q^{*} \approx 0.38$ & $H^{*} \approx 24$ & $H^{*} \approx 14$ & $H^{*} \approx 6$ \\
\hline
\end{tabular}

The large reduction in the peak pressures for either case with partial valve obstruction is visible, and as expected the smaller obstruction yielded smaller pressure peaks. The pressure peak drop ranged from a maximum of $83 \%$ for the maximum flow $\left(Q^{*} \sim 0.51\right)$ and $81 \%$ obstruction down to $42 \%$ for the minimum flow rate $\left(Q^{*} \sim 0.38\right)$ and $89 \%$ obstruction. It is important to notice in Table 4.2 that even though the flow rates that are compared are fairly similar within the same degree of obstruction, there may be more significant differences in the air pocket volumes entrapped upon valve operation, considering the sensitivity of cavity volumes to slight changes in the flow rate.

Figures 4.11 and 4.12 present the pressure hydrographs for the partial valve obstruction experiments using the intermediate flow rate $Q^{*}=0.42$ and varying slopes.

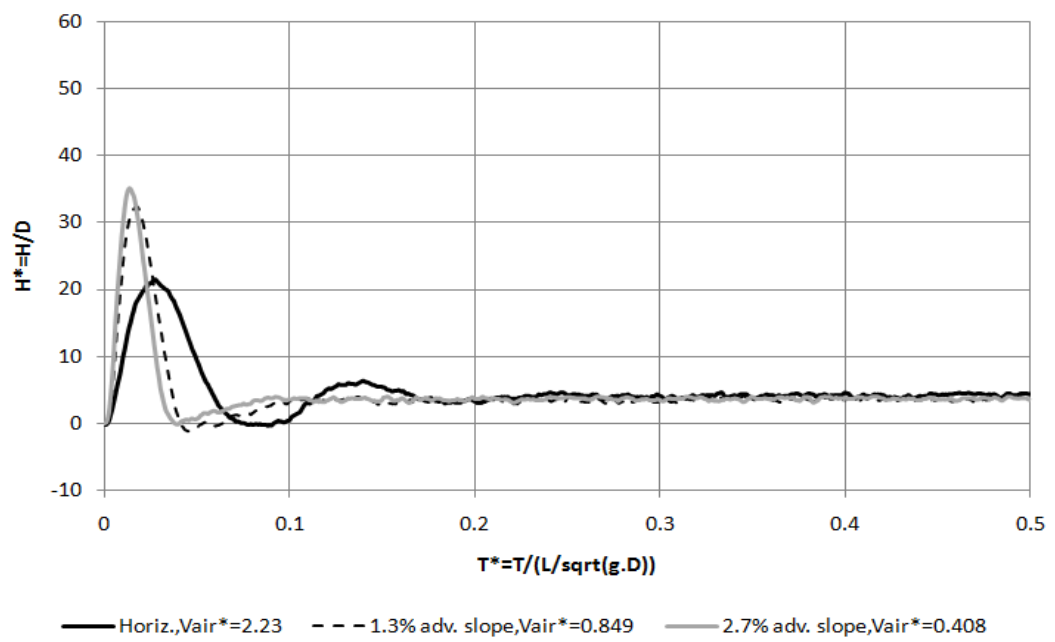

Figure 4.11 Non-dimensional pressure development following $89 \%$ obstruction by the knife gate valve $\left(Q^{*}=0.41-\right.$ 0.43 , varying slopes).

The entrapped air pocket volume for the horizontal slope was much larger than those at comparable flows at different slopes, and as a result the peak 
pressures were larger for the cases with adverse slope. Figure 4.11 presents the result for the $89 \%$ flow obstruction, and even though there was a difference in the air pocket volumes between the two adverse slope conditions, the pressure peak in both cases is similar. We have no explanation for this result. Small residual oscillations in the pressure are observed.

Figure 4.12 results are similar to the previous plot, except that it presents the result for the $81 \%$ flow obstruction for different slopes. The same trend observed in Figure 4.11 is also seen, with the horizontal slope yielding smaller pressure peaks because of the larger air pocket. The significant differences are that the discrepancy with the horizontal case is smaller than the case with $89 \%$ obstruction, and that the pressure peaks, as expected, are significantly smaller. No residual oscillations in the pressure are seen.

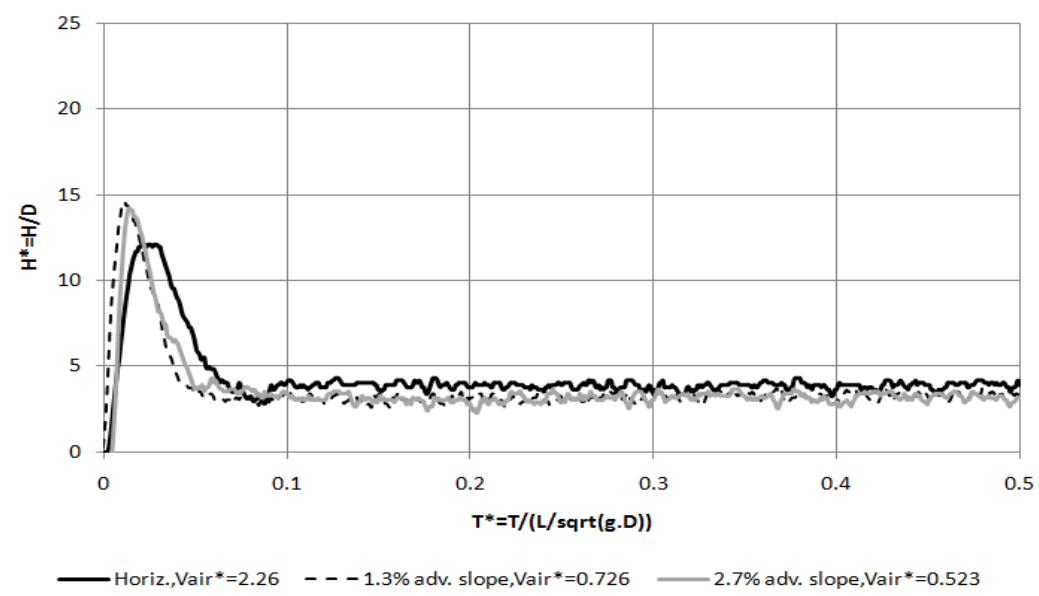

Figure 4.12 Non-dimensional pressure development following $81 \%$ obstruction by the knife gate valve $\left(Q^{*}=0.42\right.$, varying slopes).

\section{Flow Velocity Results}

Flow velocity measurements for the partial obstruction experiments reflected the pressure measurements. Upon valve operation, flow decayed rapidly and readjusted to a new steady state in under 1.2 seconds $\left(\mathrm{T}^{*}<0.1\right)$. Generally the drop in flow velocity measured by the ADV, and hence flow rate in the pipe, ranged between $50 \%$ and $64 \%$ of the initial flow rate for the case with $81 \%$ obstruction; the experiments with $89 \%$ obstruction indicated a drop of $72 \%$ to $80 \%$ of the initial flow rate. 
Figures 4.13 and 4.14 present velocity measurements for each one of those obstructions and horizontal slope, with three flow rate ranges represented.

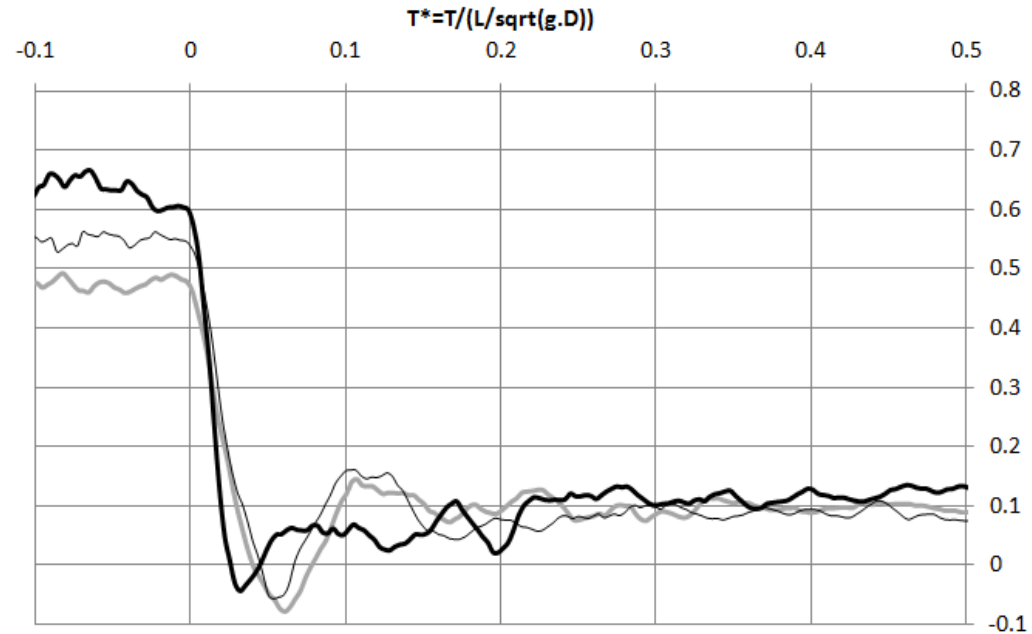

$$
\text { — } \mathrm{Q}^{*}=0.382-\mathrm{Q}^{*}=0.430-\mathrm{Q}^{*}=0.503
$$

Figure 4.13 Non-dimensional velocity development following $89 \%$ obstruction by the knife gate valve, horizontal slope results.

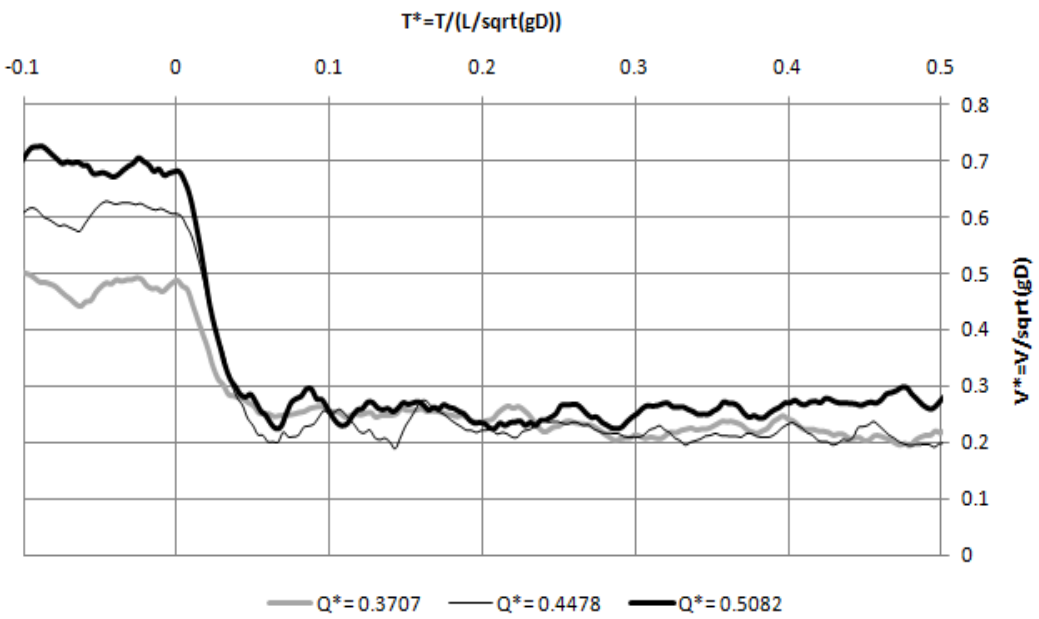

Figure 4.14 Non-dimensional velocity development following $81 \%$ obstruction by the knife gate valve, horizontal slope results. 
It is interesting to notice that, regardless of the initial flow rate, the flow measured after the valve operation was very similar between the different cases tested. One speculates that even with different available heads in the reservoirs, associated with different values of $Q^{*}$, the losses at the valve are so dominant that they offset any significant difference in flow rates.

One may also notice that for the cases with $89 \%$ obstruction there was some oscillation of the velocity, which lasted briefly. There were no noticeable oscillations in the results obtained with the $81 \%$ obstruction.

\subsection{Data Analysis and Discussion}

All peak pressure results following total or partial gate obstructions are presented in Figure 4.15. Figure 4.15a (horizontal slope results) also presents the minimum negative pressure observed in the full obstruction conditions. This graph facilitates the comparison between different air pocket volumes and obstruction degrees. Figures $4.15 \mathrm{~b}$ and $4.15 \mathrm{c}$ present measured peak pressures for adverse slopes of $1.3 \%$ and $2.7 \%$, respectively.

In agreement with the data presented in Table 4.2 , the pressure peaks resulting from the $89 \%$ obstruction are slightly less than half of those observed with total valve obstruction for similar air pocket volumes; results from $81 \%$ obstruction were about half of the values obtained with the $89 \%$ obstruction. The scatter in the results is attributed either to small discrepancies in the flow rate or air pocket volumes, or to the inability to shut the knife gate valve at the exact same time between the experimental repetitions.

A feature of Figure 4.15 is the tendency towards smaller peak pressures, with comparatively the same air pocket volume, as the slope becomes steeper. The reason for this behaviour is clarified in Figure 4.16, where the peak pressures following total obstruction are plotted as a function of the pipeline flow rate, grouped by pipeline slope.

Horizontal slopes require larger flow rates to yield comparable pressure peaks to those observed in the adverse slopes. As an example, in Figure 4.16 below, pressure peaks $H^{*}=H / D=75$ are associated with a flow rate $Q^{*}=0.50$ for the horizontal slope; for the $2.7 \%$ adverse slope this value is around $Q^{*}=0.40$. However, in terms of air pocket volume for this same pressure peak, Figure 4.15 shows that the horizontal case has an air volume around $V_{\text {air }} *=0.8$, while for the $2.7 \%$ slope the pocket volume is about $V_{\text {air }} *=0.5$. This is an indication that pressure peaks are, as expected, determined by a combination of air pocket volume and flow inertia. 

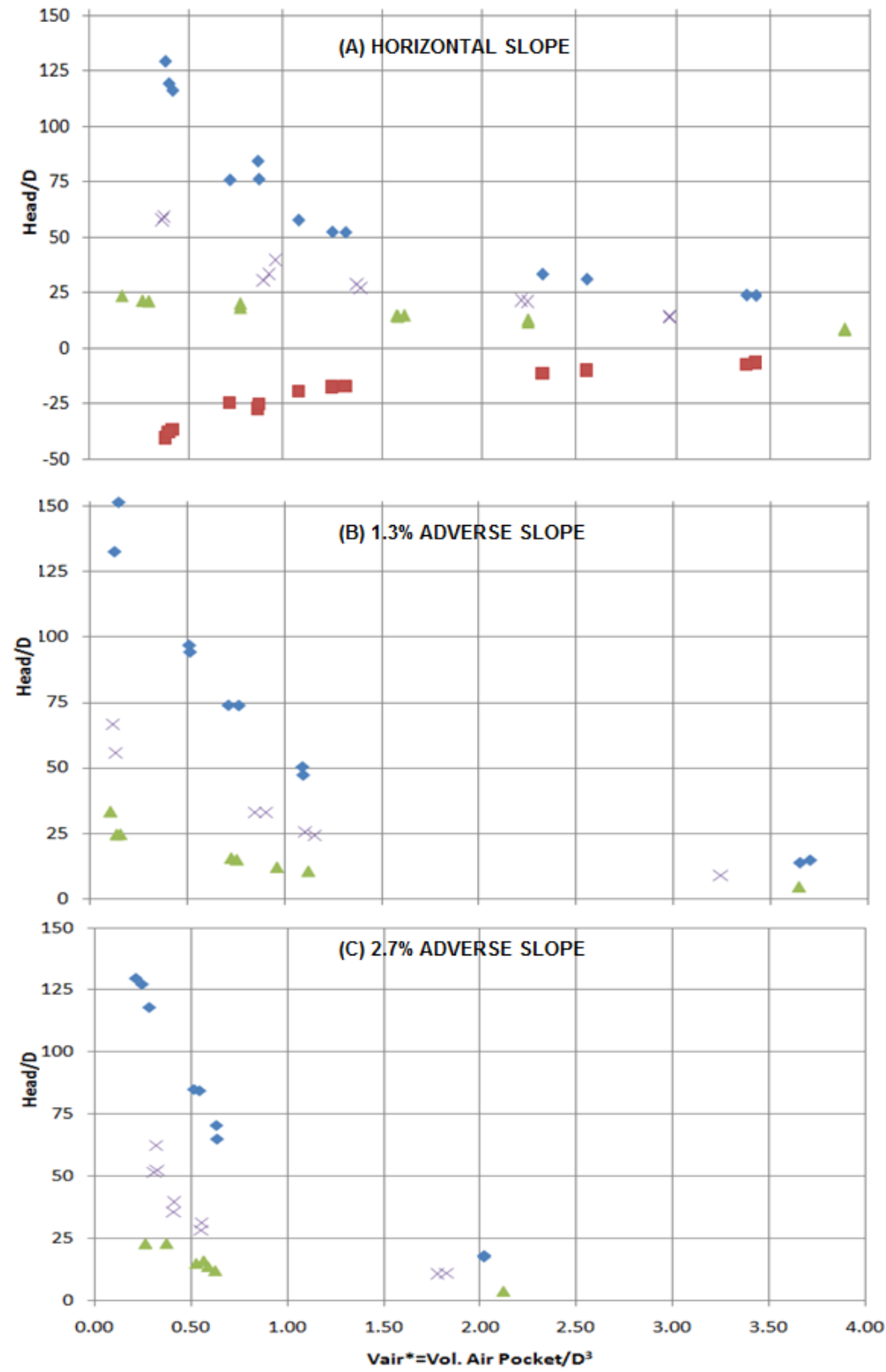

$\downarrow$ Full closure Max. Head $\square$ Full closure Min. Head $\Delta 81 \%$ closure Max. Head $\times 89 \%$ closure Max. Head

Figure 4.15 Non-dimensional peak pressures as a function of initial air pocket volume and pipeline slope. 


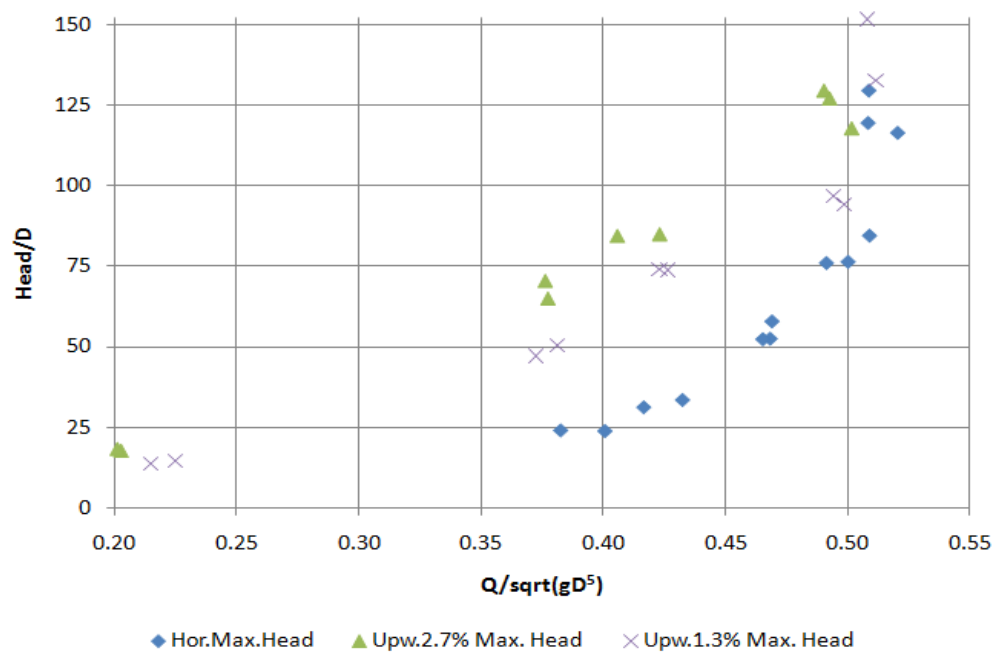

Figure 4.16 Non-dimensional peak pressures as a function of initial flow rate and slope for total obstruction experiments.

The ratio between the peak pressures measured at $X^{*}=0.5$ and the peak pressure measured at the downstream end $X^{*}=1.0$ is presented in Figure 4.17 for all tested cases, as a function of the air pocket volume. Despite of the data scatter, it noticeable that for smaller air pocket volumes the pressure peak ratio ranged between 0.55 and 0.75 , while this ratio had a tendency to increase for larger air pocket volumes to values above 0.85 .

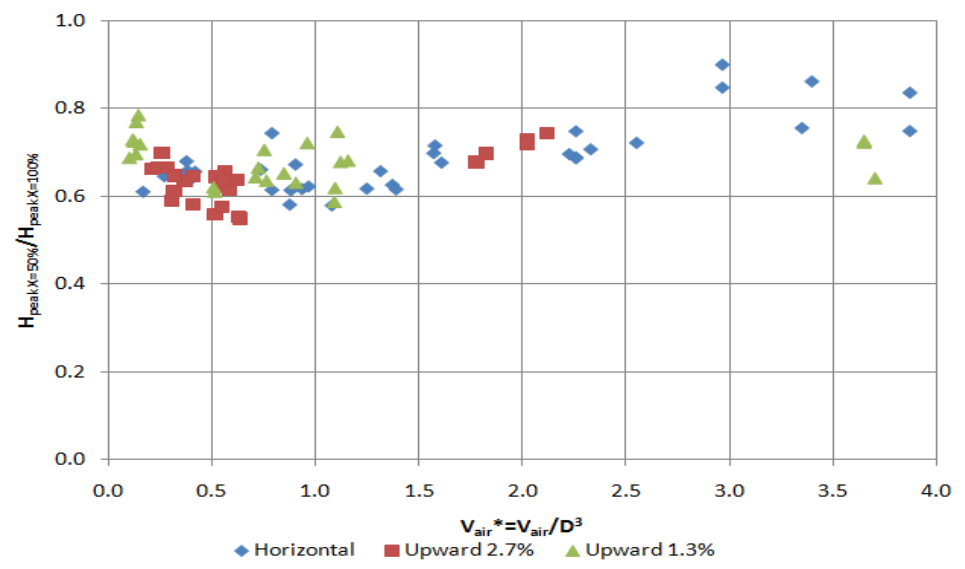

Figure 4.17 Ratio between peak pressures measured at $X^{\star}=0.5$ and $X^{*}=1.0$ as a function of the entrapped air pocket volume. 


\subsection{General Comments and Future Work}

Surging in stormwater tunnels is an extremely important issue because of potential operational problems. To date our understanding of this problem is still limited, particularly with respect to the influence of air. It is understood that air compressibility may create conditions with high peak pressures, and many previous studies focused in determining an estimate of what would be the maximum pressure in tunnel systems. Such maximum pressures are associated with conditions where no air escape is allowed during compression; the subsequent minimum pressures are also significant, in some cases well under atmospheric values. The assumption that these conditions are representative for design purposes may result in unrealistic structural requirements, leading to more expensive construction costs than necessary.

It is estimated that nearby vertical shafts provide surge relief during the airwater compression and expansion phases, which in turn may significantly alter the system dynamics. If this relief is properly considered, better estimates of upsurge and downsurge pressures should be available, leading to more realistic structural requirements and construction costs for tunnels. However, to date no experimental study in stormwater tunnel surging has considered this. Albeit an idealization, the experiments with partial closure presented here offer a first glimpse of how different are the results when pressure relief (presently in the form of an escaping water mass during the surging phase) is provided.

One important finding in the research to date is the great difference in flow characteristics between total and partial flow obstructions. The experiments performed here with complete gate obstruction conditions present results with the general characteristics of a spring mass system, with pressure oscillations that have higher magnitude and frequencies for smaller air pockets.

On the other hand, partial obstruction experiments, which aimed to create some pressure relief during the air pocket compression events, yielded very different results, in a pattern that has not been identified in previous investigations. The single pressure pulse behaviour is very different in nature from the spring mass system in that the pressure magnitudes are significantly smaller, the negative pressures are much diminished, and pressure oscillations, when observed, are much more rapidly damped. It is very important that this behaviour has been identified, so that numerical tools may be constructed to incorporate those observations.

This research also has been useful in helping to isolate the role of the initial system's inertia and the air pocket volume in the measured pressure surges following valve operation. This was possible because experiments with comparable pocket volumes were performed with significantly different initial flow rates due to the different pipe slopes used. It was confirmed that the larger 
pressure peaks would be associated with larger initial inflow rates, even in pockets of comparable sizes. Previous studies in air pocket compression did not involve the variation of water flow rates, so these findings are also very useful in the calibration of future numerical models.

The next phase of experiments will focus in favourable slope experiments, in which it is expected that much larger flows will sustain the air pockets in the same volume range tested so far. In such conditions the behaviour of pressure peaks when there are much larger air pocket volumes and flow rates still needs to be investigated. Moreover, this phase will allow for further observation of the shape of entrapped air pockets, how they are distributed, and how they move following the passage of pipe filling bores.

The observation of the pocket motion and redistribution following entrapment and the study of air entrainment caused by sweeping pipe filling bores are possibly the most relevant topics to future studies. The ability to simulate the pressure peaks with lumped inertia models is a good indication that discretized models (e.g. based on the solution of the Saint-Venant equations) can be adapted to handle similar conditions. However, air pockets do migrate, and this may affect the overall prediction is not properly accounted for in the computations. This should be included in subsequent research.

\subsection{Acknowledgments}

The authors gratefully acknowledge the support by LimnoTech, Inc. and Auburn University of the present work.

\section{References}

Fuertes, V. S., Arregui, F., Cabrera, E. and Iglesias, P. L. (2000). "Experimental setup of entrapped air pockets model validation". Proc. 8th International Conference on Pressure Surges - Safe Design and Operation of Industrial Pipe Systems, BHRA, The Hague, Netherlands, pp. 133-145.

De Martino, G., Fontana N. and Giugni, M. (2008). Transient Flow Caused by Air Expulsion through an Orifice". J. Hydr. Engrg., 134(9):1395-1399.

Guo, Q. and Song, C. S. S. (1991). "Dropshaft hydrodynamics under transient conditions.” J. Hydr. Engng., 117(8), 1042-1055.

Hamam, M. A. and McCorquodale, J. A. (1982). "Transient conditions in the transition from gravity to surcharged sewer flow.” Can. J. Civ. Engrg., (9),189-196.

Leite, G.M. and J. Vasconcelos. 2011. "Numeric Modeling of Water Mains Filling Considering Air Pressurization." Journal of Water Management Modeling R241-01. doi: 10.14796/JWMM.R241-01.

Li J. and McCorquodale, A. (1999). "Modeling mixed flow in storm sewers". J. Hydr. Engrg., 125(11):1170-1180. 
Martin C. S. (1976). "Entrapped air in pipelines”. In BHRA, Proc. 2nd Int. Conf. Pressure Surges, Bedford, England.

Vasconcelos J. G. and Wright, S. J. (2005). "Experimental investigation of surges in a stormwater storage tunnel" J. Hydr. Engrg, 131(10):853-861.

Vasconcelos J. G. and S. J. Wright, S. J. (2006). "Mechanisms for Air pocket Entrapment in Stormwater Storage Tunnels" Proc. 2006 ASCE EWRI Congress, Omaha, NE.

Wright, S. J., Lewis, J. W. and Vasconcelos, J.G., (2011).”Geysering in Rapidly Filling Storm-Water Tunnels". J. Hydr. Engng. Vol. 137(1) 112-115.

Zhou, F., Hicks, F. E. and Steffler P. M. (2002a) "Transient flow in a rapidly filling horizontal pipe containing trapped air". J. Hydr. Engrg., 128(6):625-634.

Zhou, F., Hicks, F. E. and Steffler, P. M. (2002b). "Observations of air-water interaction in a rapidly filling horizontal pipe.” J. Hydr. Engrg., ASCE, 128(6), 635-639. 